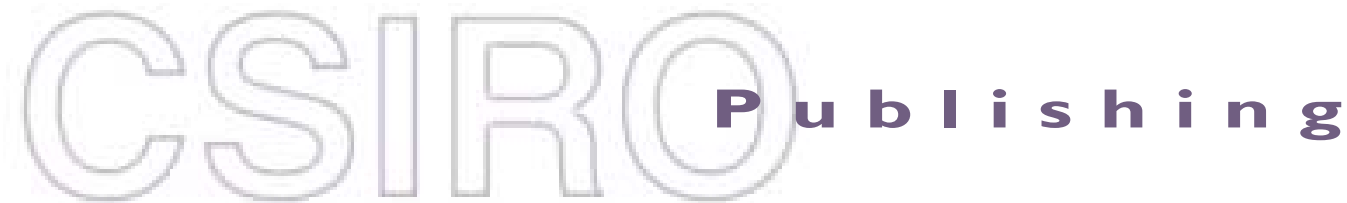

\section{Publications of the Astronomical Society of Australia}

Volume 19, 2002

(C) Astronomical Society of Australia 2002

An international journal of astronomy and astrophysics

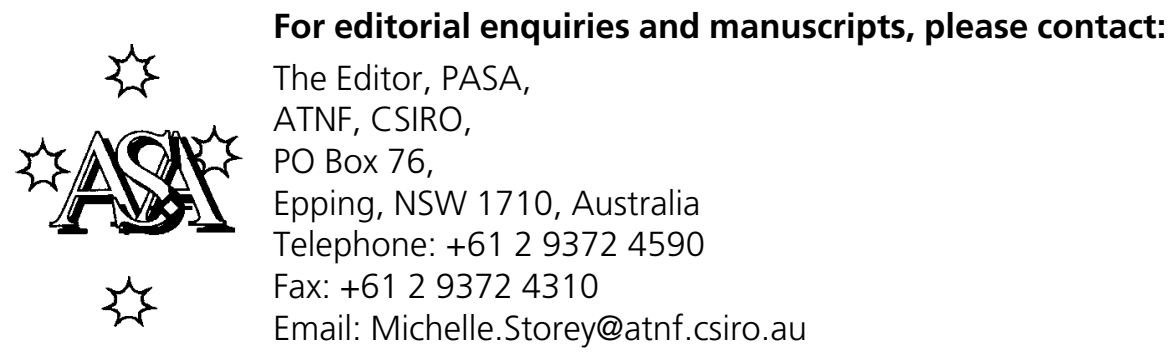

For general enquiries and subscriptions, please contact: CSIRO Publishing PO Box 1139 (150 Oxford St)

Collingwood, Vic. 3066, Australia

Telephone: +6139662 7666

Fax: +61 396627555

Email: publishing.pasa@csiro.au

C S I RO

PUBLISHING Published by CSIRO Publishing

for the Astronomical Society of Australia

www.publish.csiro.au/journals/pasa 


\title{
Near Infrared Micro-variability of Radio-loud Quasars
}

\author{
Matthew Whiting, Alicia Oshlack and Rachel Webster \\ Astrophysics Group, School of Physics, University of Melbourne, Victoria 3010, Australia \\ mwhiting@physics.unimelb.edu.au \\ aoshlack@physics.unimelb.edu.au \\ rwebster@physics.unimelb.edu.au
}

Received 2001 April 4, accepted 2001 November 5

\begin{abstract}
We observed three AGN from the Parkes Half-Jansky Flat-spectrum Sample at near infrared (NIR) wavelengths to search for micro-variability. In one source, the blue quasar PKS 2243-123, good evidence for NIR micro-variability was found. In the other two sources, PKS 2240-260 and PKS 2233-148, both BL Lacertae objects, no such evidence of variability was detected. We discuss the implications of these observations for the various mechanisms that have been proposed for micro-variability.
\end{abstract}

Keywords: quasars: individual (PKS 2243-123) - BL Lacertae objects: individual (PKS 2240-260, PKS 2233-148)

\section{Introduction}

One of the distinctive features of blazar AGN is rapid flux variability, which is seen in every region of the electromagnetic spectrum (Wagner \& Witzel 1995). This variability can be on timescales as short as hours to minutes - in this case, it is termed 'micro-variability'. In the optical, these variations can be of the order of $\sim 0.1$ mag per night (see the review by Miller \& Noble 1996). While some radio-quiet quasars have been shown to exhibit microvariability (Jang \& Miller 1997), it is seen most commonly in radio-loud (mostly blazar-type) AGN. The duty cycle ${ }^{1}$ for radio-loud AGN is $\sim 68 \%$ (Romero, Cellone, \& Combi $1999)$ and the figure may be even higher $(\sim 80 \%)$ for radio-selected BL Lacs (Heidt \& Wagner 1996).

For radio-quiet AGN, the situation is less clear. Romero et al. (1999) found a low duty cycle of $\sim 7 \%$. GopalKrishna et al. (2000), in their extensive study of microvariability of radio-quiet QSOs, found that about $31 \%$ of the objects showed evidence for variability. They suggest that radio-quiet QSOs are less likely to exhibit microvariability in a given time span than radio-loud AGN, and that the character of the variations in the two classes tend to be different. However, de Diego et al. (1998), in a comparative study of radio-loud and radio-quiet quasars found that micro-variations may be as common in radio-quiet quasars as radio-loud quasars. While the micro-variations in radio-loud objects are thought to be due to shocks within the jet, the existence of similar variability in radioquiet objects indicates that the accretion disk may also be important in producing the variations.

We are conducting an on-going study of the optical and near infrared emission from objects from the Parkes HalfJansky Flat-spectrum Sample (PHFS, Drinkwater et al. 1997), with the data and modelling already presented

\footnotetext{
${ }^{1}$ The fraction of time that an object in a given class is variable. This figure is determined observationally by taking the ratio of the time for which objects of a given class are variable to the total observing time for objects in that class.
}

in Francis, Whiting, \& Webster (2000, hereafter FWW) and Whiting, Webster, \& Francis (2001, hereafter WWF) respectively. Based on the above estimates for microvariability duty cycles, it is reasonable to expect to see micro-variability in quasars and BL Lacs from the PHFS. This paper contains an investigation into the existence of micro-variability in three such sources. We chose three objects from the PHFS to search for micro-variability at near infrared wavelengths - a wavelength region that has rarely been used for such a study. With the above figures, if three objects are observed there is a probability of $\gtrsim 97 \%$ of seeing micro-variability in at least one source. We also chose objects with different optical/NIR spectra, so that we have both jet-dominated and accretion disk-dominated sources. This will enable a comparison of the two ideas for the production of micro-variability.

\section{Observations}

On the night of August 3, 1999, three AGN from the PHFS were observed multiple times in three NIR filters over the course of $\sim 7.5 \mathrm{~h}$, in an attempt to detect short timescale micro-variability. The observations were made in the NIR with the CASPIR $256 \times 256 \mathrm{InSb}$ array camera (McGregor et al. 1994) on the Australian National University's $2.3 \mathrm{~m}$ telescope, at Siding Spring Observatory, NSW. The images were taken using the fast camera with $0.5^{\prime \prime} /$ pixel, and thus the entire detector spans a little over 2 arcmin.

The three objects chosen were the following:

- PKS 2243-123: A quasar, $z=0.63$, that was described as optically variable by Shen et al. (1998), although they did not give a reference for this classification (it possibly comes from the description as variable in Hewitt \& Burbidge (1993)). It has a blue optical/ NIR spectral energy distribution (SED) from FWW, with $B-K=2.15$. WWF modelled the SEDs of many PHFS sources by fitting a combination of a blue 

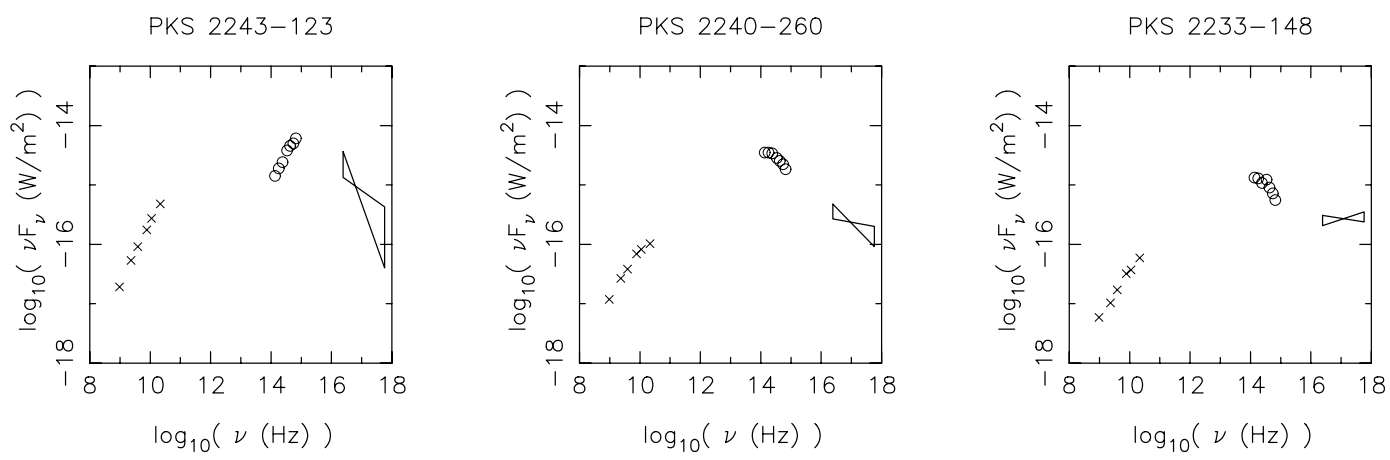

Figure 1 Plots of the multi-wavelength SED for each of the sources observed. The radio data (crosses) come from Kovalev et al. (1999), the optical/NIR photometry (circles) is from Francis et al. (2000), and the X-ray observation is from Siebert et al. (1998).
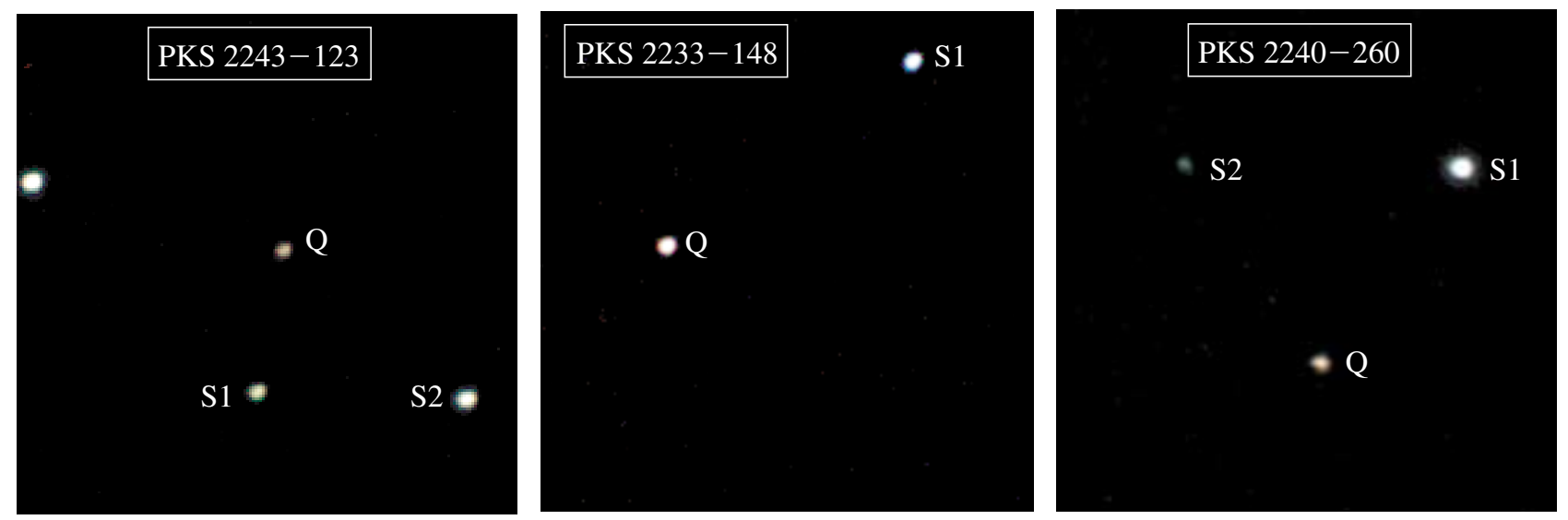

Figure 2 Three-colour composite NIR images of the sources used for the micro-variability analysis. The source in question is indicated by a 'Q', while the comparison stars (discussed in Section 3) are indicated by 'S1' and 'S2'. In all images, north is up and east is to the left.

power law plus a synchrotron model with a turn-over. This source was best fitted with a power law of the form $\lambda^{-1.92}$, and showed no evidence for synchrotron emission in its optical/NIR SED. The optical spectrum of the source shows strong broad Balmer lines and [O III] lines of small equivalent width (Tadhunter et al. 1993).

- PKS 2240-260: A BL Lac object at $z=0.774$. It has a redder optical/NIR SED $(B-K=4.43$ from FWW, and is fitted by WWF with a strong synchrotron component). High optical polarisation has been observed in this source (15.1\%, Wills et al. (1992)), and only weak, if any, emission lines have been seen. This object was seen to exhibit optical intra-day variability by Heidt \& Wagner (1996).

- PKS 2233-148: A candidate for a BL Lac (according to Padovani \& Giommi (1995)), at $z>0.609$. (The redshift of $z=0.325$ quoted in Johnston et al. (1995) and on $\mathrm{NED}^{2}$ appears to be due to a misreading of a table in Schmidt \& Green (1983).) This has a similar optical/NIR SED to PKS 2240-260, also dominated by synchrotron emission.

The multi-wavelength (radio-X-ray) SED for each source is shown in Figure 1, while three-colour images

${ }^{2}$ The NASA/IPAC Extragalactic Database, http://nedwww.ipac.caltech. edu/ of each of the sources are shown in Figure 2, indicating the location of the source, as well as nearby stars that were used in the analysis (see Section 3 for details).

These sources were chosen primarily on the basis of their position on the sky (to enable constant observations for the second half of a night, and to minimise the time required for the telescope to move between each source), and also to examine the potential for micro-variability in both BL Lacs (PKS 2240-260 and PKS 2233-148) and blue radio-loud quasars (PKS 2243-123).

These objects were observed in three filters: $J, H$, and $K_{n}$. Each source was observed eight times in each filter. Each of the images is comprised of $2 \times 2$ dithered $60 \mathrm{sec}$ images, each made up of 12 averaged $5 \mathrm{sec}$ exposures in $K_{n}$, six averaged $10 \mathrm{sec}$ exposures in $H$, and two averaged $30 \mathrm{sec}$ exposures in $J$. The flat field was created from the difference of dome exposures with the lamps on and off - this removes any telescope emission, improving greatly the photometric accuracy. The sky emission was removed by using a median of the four dithered images of the same band taken at the same time. The four images were then aligned and combined using the median (to remove any residual errors). The photometry was done with the apphot package in IRAF, using circular apertures with the sky background level determined by the median flux in an annulus around the source. Several different sizes of aperture were used to make sure the underlying 


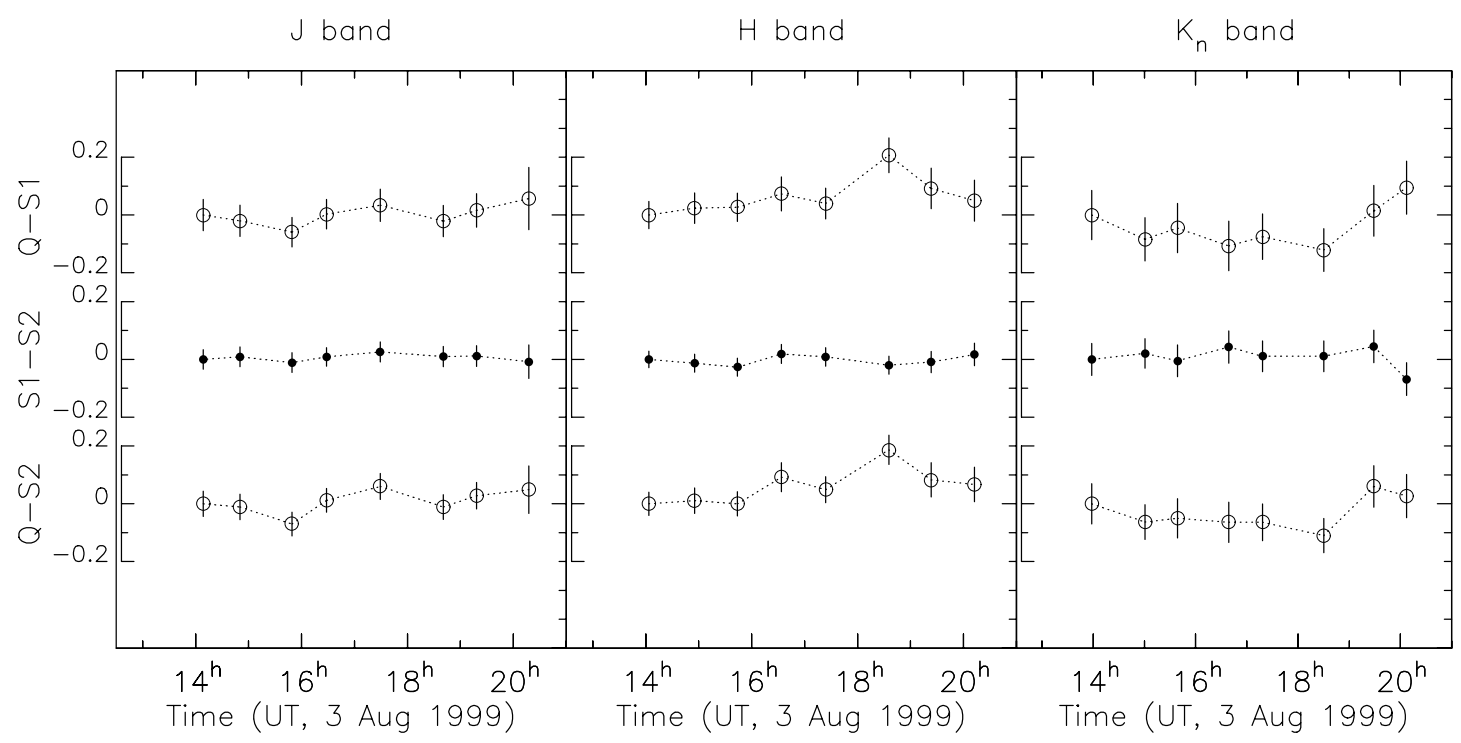

Figure 3 Differential light-curves for PKS 2243-123. Shown are the quasar-star light-curves for each comparison star (Q-S1 and Q-S2), as well as the star-star comparison light-curve (S1-S2). The error bars are just the photometric errors. The vertical scales show tick marks every $0.1 \mathrm{mag}$, with the zero point for each curve set at the first observed point. The true values of these initial points are given in Table 1 .

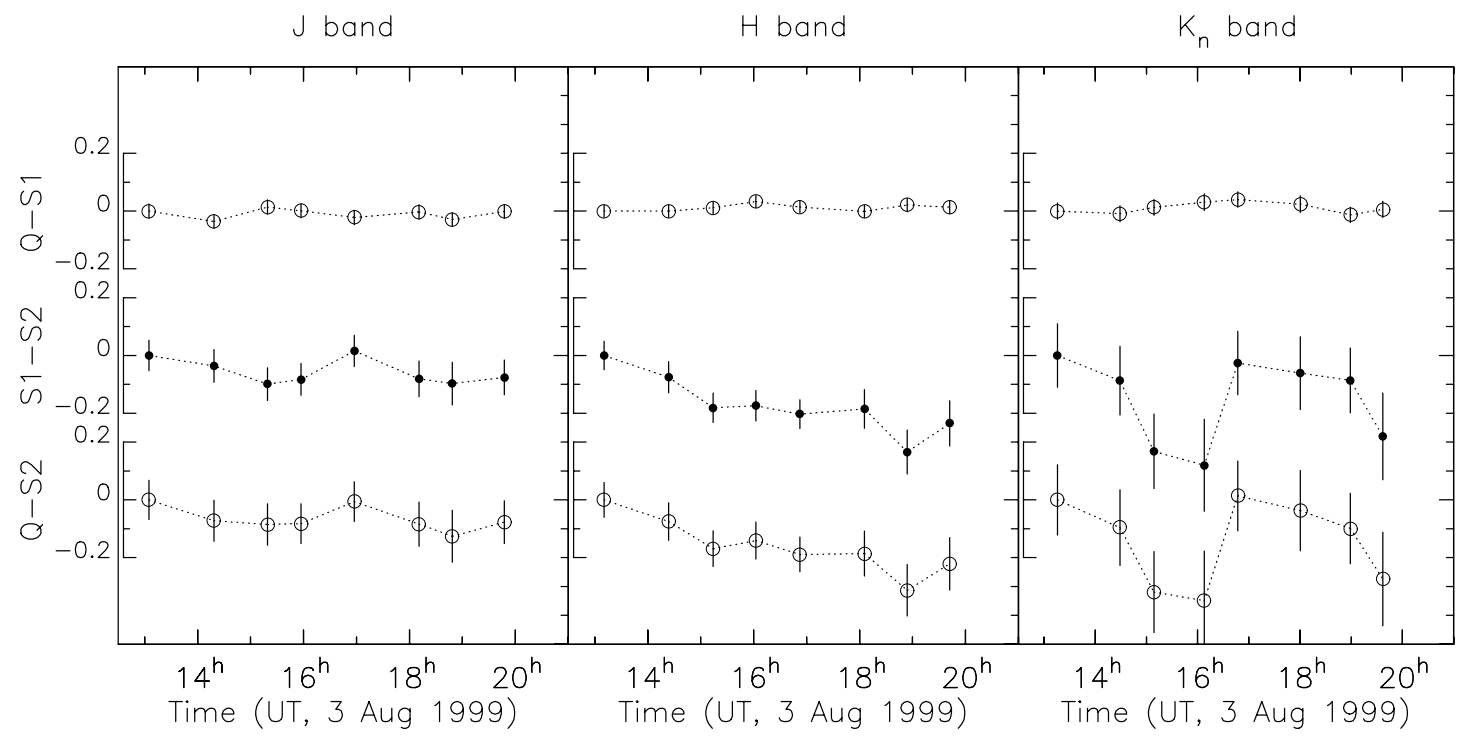

Figure 4 Differential light-curves for PKS 2240-260. Details are as for Figure 3.

host galaxy was not affecting the observed variability. No significant difference was seen for the different aperture sizes - the results presented here use an aperture of $5^{\prime \prime}$. The seeing varied from $\sim 1-1.2^{\prime \prime}$ over the course of the night. The errors in the photometry shown on the lightcurves are purely the random errors from the photometry calculations.

\section{Measuring the Variability}

In order to construct light-curves for each of the sources, the differential magnitude of the quasar is measured relative to comparison stars in the same field. This differential magnitude is given by $\Delta m=m_{q}-m_{c}$, where $m_{q}$ and $m_{c}$ are the magnitudes of the quasar and the comparison star respectively. This differential magnitude is then compared to the comparison differential magnitude $\Delta m^{\prime}=m_{c}-m_{c^{\prime}}$, where $m_{c^{\prime}}$ is the magnitude of a second comparison star in the same field. This second differential magnitude acts as a check - a change in flux in the quasar will change $\Delta m$ but not $\Delta m^{\prime}$, while a change that occurs in both will not be intrinsic to the quasar. The light-curves are presented in Figures 3-5. For clarity, they are offset from each other and plotted with their own vertical scale, relative to the initial point. The zero points for these scales are given in Table 1.

It must be noted here that PKS 2233-148 did not have a second comparison star in the small $\left(\sim 2^{\prime}\right)$ field of view 


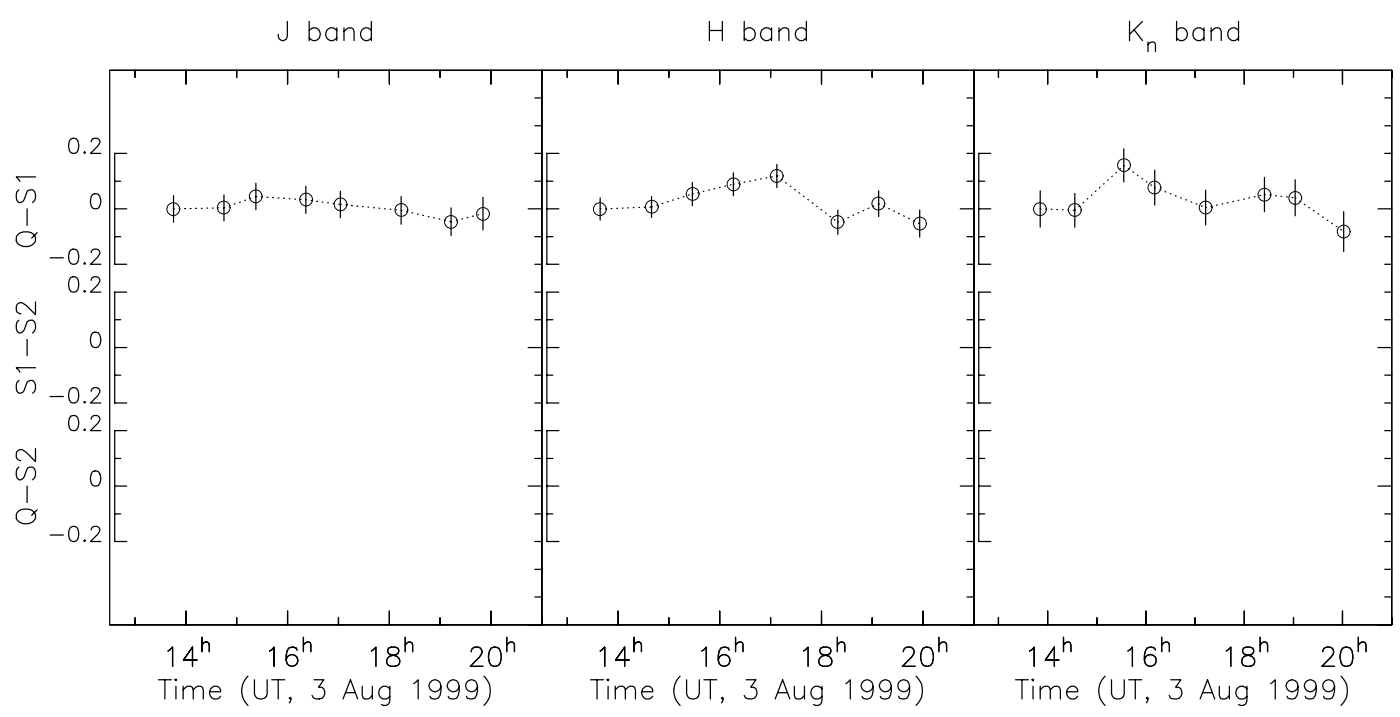

Figure 5 Differential light-curves for PKS 2233-148. Details are as for Figure 3.

Table 1. Offsets for the micro-variability light-curves presented in Figures 3-5. To obtain the correct differential magnitude for each light-curve, add the corresponding number from the table to the values in the figure

\begin{tabular}{lcrrr}
\hline Source & Band & Q-S1 & Q-S2 & S1-S2 \\
\hline PKS 2243-123 & $J$ & 0.65 & 1.86 & 1.21 \\
PKS 2243-123 & $H$ & 0.79 & 1.73 & 0.93 \\
PKS 2243-123 & $K_{n}$ & 0.51 & 1.32 & 0.81 \\
PKS 2240-260 & $J$ & 2.61 & -1.44 & -4.05 \\
PKS 2240-260 & $H$ & 2.16 & -1.41 & -3.58 \\
PKS 2240-260 & $K_{n}$ & 1.49 & -2.12 & -3.61 \\
PKS 2233-148 & $J$ & 0.37 & - & - \\
PKS 2233-148 & $H$ & 0.07 & - & - \\
PKS 2233-148 & $K_{n}$ & -0.56 & - & - \\
\hline
\end{tabular}

of CASPIR. This means that the full variability analysis cannot be done for this source. However, a light-curve for $\Delta m$ is presented (Figure 5), using the one available star.

To determine whether a given source is variable, we use the $99 \%$ criterion from Jang \& Miller (1997) and Romero et al. (1999). We define the variability confidence level $C=\sigma_{T} / \sigma$, where $\sigma_{T}$ and $\sigma$ are the standard deviations of the target (quasar-star) and comparison (star-star) lightcurves. The variability criterion requires that, for a source to be variable, $C>2.576$. Note that these standard deviations merely come from the point-to-point fluctuations in the light curve, and bear no relationship to the error bars displayed in Figures 3-5.

If a source is found to be variable, we also define (in a manner similar to that of Romero et al. (1999)) the variability amplitude of each light-curve by

$$
A=\sqrt{\left(D_{\max }-D_{\min }\right)^{2}-2 \sigma^{2}},
$$

where $D_{\min }$ and $D_{\max }$ are the minimum and maximum of the differential light-curve respectively. Unlike the amplitude defined in Romero et al. (1999), this amplitude is measured in magnitudes, and thus avoids the dependence that a relative amplitude (i.e. as a percentage) has on the normalisation of the light-curve. Table 2 shows the values for $\sigma_{T}$ for each source with respect to each comparison star, as well as the confidence level $C$ and, when the source is variable, the amplitude $A$ of that light-curve.

\section{Conclusions}

\subsection{Results}

NIR micro-variability is detected in one source, PKS 2243-123. There is significant variability in both $J$ and $H$ bands, while at $K_{n}$ band, despite both the Q-S curves showing variability (with a greater $\sigma_{T}$ than the other two bands), there is also significant noise present in the comparison light-curve, making it impossible to say at $99 \%$ confidence that the quasar itself is varying. It is also worth noting that we see different variability patterns in the different bands (e.g. compare the $H$ and $K_{n}$ light-curves). A possible cause for this is very fast timescale flickering that affects the different bands differently (since the observations for the various bands are not exactly simultaneous). These observations, nonetheless, demonstrate the additional information acquired when multi-colour data are used in variability analysis (something that is rarely done).

The results for the other two sources are more equivocal. Firstly, the lack of a second comparison star for PKS 2233-148 means no conclusions can be drawn about the significance of its fluctuations, although we do note that the values of $\sigma_{T}$ in the $H$ and $K_{n}$ bands are comparable to those of PKS 2243-123.

Secondly, the analysis of PKS 2240-260 is compromised by comparison star 2 . Since both the Q-S2 and $\mathrm{S} 1-\mathrm{S} 2$ curves show the same variability, it is clear that the origin of this variability is with this star, and not the BL Lac. However, even the Q-S1 light-curve shows little variability, with the $\sigma_{T}$ values being less than half those of PKS 2243-123, indicating that the source is most likely 
Table 2. Micro-variability results for the three sources. The standard deviation is of the comparison curves of the quasar with respect to each of the comparison stars, in each band. The values of the variability confidence level $C$ are shown where there is more than one comparison star, and the variability amplitude $A$ is shown for the cases where $C>\mathbf{2 . 5 7 6}$

\begin{tabular}{|c|c|c|c|c|c|c|}
\hline Source name & Band & $\sigma(\mathrm{mag})$ & Comparison star & $\sigma_{T}(\mathrm{mag})$ & $C$ & $A$ (mag) \\
\hline \multirow{2}{*}{ PKS 2243-123 } & $J$ & 0.011 & 1 & 0.048 & 4.56 & 0.15 \\
\hline & & & 2 & 0.053 & 4.99 & 0.18 \\
\hline \multirow[t]{2}{*}{ PKS 2243-123 } & $H$ & 0.017 & 1 & 0.063 & 3.59 & 0.21 \\
\hline & & & 2 & 0.066 & 3.77 & 0.21 \\
\hline \multirow[t]{2}{*}{ PKS 2243-123 } & $K_{n}$ & 0.052 & 1 & 0.100 & 1.90 & $(0.28)$ \\
\hline & & & 2 & 0.071 & 1.37 & $(0.24)$ \\
\hline \multirow[t]{2}{*}{ PKS 2240-260 } & $J$ & 0.081 & 1 & 0.022 & 0.27 & - \\
\hline & & & 2 & 0.092 & 1.13 & - \\
\hline \multirow[t]{2}{*}{ PKS 2240-260 } & $H$ & 0.125 & 1 & 0.015 & 0.12 & - \\
\hline & & & 2 & 0.121 & 0.97 & - \\
\hline \multirow[t]{2}{*}{ PKS 2240-260 } & $K_{n}$ & 0.173 & 1 & 0.026 & 0.15 & - \\
\hline & & & 2 & 0.168 & 0.97 & - \\
\hline PKS 2233-148 & $J$ & - & 1 & 0.023 & - & - \\
\hline PKS 2233-148 & $H$ & - & 1 & 0.064 & - & - \\
\hline PKS 2233-148 & $K_{n}$ & - & 1 & 0.065 & - & - \\
\hline
\end{tabular}

Table 3. Micro-variability results for the second comparison star in the PKS 2240-260 field

\begin{tabular}{lcccccc}
\hline Source name & Band & $\sigma(\mathrm{mag})$ & Comparison object & $\sigma_{T}(\mathrm{mag})$ & $C$ & $A(\mathrm{mag})$ \\
\hline PKS 2240-260 S2 & $J$ & 0.022 & $\mathrm{Q}$ & 0.092 & 4.22 & 0.34 \\
& & & $\mathrm{~S} 1$ & 0.081 & 3.72 & 0.30 \\
PKS 2240-260 S2 & \multirow{2}{*}{0.015} & $\mathrm{Q}$ & 0.121 & 8.21 & 0.44 \\
& \multirow{2}{*}{$K_{n}$} & \multirow{2}{*}{0.026} & $\mathrm{~S} 1$ & 0.125 & 8.45 & 0.45 \\
PKS 2240-260 S2 & & $\mathrm{Q}$ & $\mathrm{S} 1$ & 0.168 & 6.50 & 0.43 \\
& & & & 0.173 & 6.70 & 0.44 \\
\hline
\end{tabular}

not varying. Since this source was observed to be variable by Heidt \& Wagner (1996), our observation indicates that we caught the source in a non-variable period. We do note however that the data from Heidt \& Wagner (1996) consisted of 41 observations over 6.39 days, so they were more likely to observe variability.

We can perform a similar analysis on star 2 (which has the designation 0600-44255475 in the USNO-A2.0 Catalogue $^{3}$ ), to quantify the degree of its variability. The results of this analysis are given in Table 3, with the Q-S1 curve acting as the comparison light-curve (i.e. the source of the $\sigma$ value). Clearly, this object is exhibiting strong micro-variability. What kind of source is this? We took a noisy spectrum of this source with the ANU $2.3 \mathrm{~m} \mathrm{DBS}$, and it indicates the source is roughly a late K-type star. This is consistent with the colours of the star in both the optical and NIR (taken from the images from FWW). We do note that it is thus too red to be an RR Lyrae star, which would have accounted for the rapidity of its variations. We tentatively suggest it is instead a flare star.

\footnotetext{
${ }^{3}$ See http://vizier.u-strasbg.fr/vizier/VizieR/pmm/usno2.htx
}

\subsection{Interpretation}

The origin of optical and NIR micro-variability in blazar AGN is not very well understood. There are two main types of models that have been developed to explain the observations. Firstly, there are models that use a shock-injet setup. This type of model has been used to explain the radio variability seen in blazars (Marscher \& Gear 1985). To produce the optical micro-variability, a thin relativistic shock within a jet encounters a feature, such as a bend in the jet or an inhomogeneity in the density, which causes an enhancement of the observed jet emission (see, for example, Gopal-Krishna \& Wiita (1992) or Qian et al. (1991)).

A second suggestion for the cause of micro-variability moves away from the jet and looks instead at the accretion disk. Perturbations or instabilities on the surface of an accretion disk (such as 'hot-spots' — regions of high temperature and/or density) can create micro-variations in the emission (see Mangalam \& Wiita (1993) and references therein). It is likely that both models are important for micro-variability in AGN, and the relative importance for individual sources depends on the relative strengths of the jet and disk emission. 
The quasar PKS 2243-123 has a blue optical/NIR SED (the photometry from these observations gives the same NIR slope as the photometry presented in FWW, and is modelled in WWF by a blue power law). It thus shows no evidence for synchrotron emission in its optical spectrum (a fact also supported by its observed strong emission lines). Hence, it is unlikely that fluctuations in the jet emission will cause the observed micro-variations, unless the jet is present at a very low level and experiences relatively large fluctuations. It seems then that the microvariability in this source is more likely to be explained by the accretion disk instability model.

To test this statement, we can take the model fitting from WWF to an extreme, so that a synchrotron component is fitted along with a very blue $\left(f_{v} \propto v^{-0.05}\right)$ power law. This generates the maximum amount of synchrotron flux according to this model. The amount of synchrotron as a proportion of total flux in the near infrared ranges from $3.4 \%$ (at $J$ band) to $5.4 \%$ (at $K_{n}$ band). If we assume that the synchrotron component is the only component that is varying, then to produce the observed fluctuations, the synchrotron component must vary in flux by a factor of at least 7 (on a timescale of about an hour). The accretion disk instability model allows us to avoid such extreme variability, and is consistent with observations of microvariability in radio-quiet quasars (e.g. Gopal-Krishna et al. 2000).

Finally, since the observations presented here were limited in scope (due to the time available), a follow-up study would be quite worthwhile. Such a study should involve selecting a small sample of quasars (and BL Lacs) that has some sources with synchrotron dominated SEDs (this part of the sample would contain the BL Lacs, based on the modelling in WWF), and some with blue power law SEDs (in the manner of PKS 2243-123). The observations would ideally cover a greater period of time than that described in this paper, to increase the probability of seeing variability. This would also enable a better determination of the nature and structure of the variability. The relative amounts of micro-variability in the two types of sources could then be gauged accurately, particularly in light of our detection of micro-variability in the blue quasar. This would hopefully give an indication of how important each model is for micro-variability in all types of radio-loud AGN.

\section{Acknowledgements}

We wish to thank the two anonymous referees for their helpful comments, and the MSSSO TAC for granting us the time on the $2.3 \mathrm{~m}$ telescope.

\section{References}

de Diego, J. A., et al. 1998, ApJ, 501, 69

Drinkwater, M. J., et al. 1997, MNRAS, 284, 85

Francis, P. J., Whiting, M. T., \& Webster, R. L. 2000, PASA, 17, 1, 56 (FWW)

Gopal-Krishna \& Wiita, P. J. 1992, A\&A, 259, 109

Gopal-Krishna, et al. 2000, MNRAS, 314, 815

Heidt, J., \& Wagner, S. J. 1996, A\&A, 305, 42

Hewitt, A., \& Burbidge, G. 1993, ApJSS, 87, 451

Jang, M., \& Miller, H. R. 1997, AJ, 114, 565

Johnston, K. J., et al. 1995, AJ, 110, 880

Kovalev, Y. Y., et al. 1999, A\&AS, 139, 545

Mangalam, A. V., \& Wiita, P. J. 1993, ApJ, 406, 420

Marscher, A. P., \& Gear, W. K. 1985, ApJ, 298, 114

McGregor, P., Hart, J., Downing, M., Hoadley, D., \& Bloxham, G 1994, in Infrared Astronomy with Arrays: The Next Generation, ed. I. S. McLean (Dordrecht: Kluwer), p. 299

Miller, H. R., \& Noble, J. C. 1996, in Blazar Continuum Variability, ASP Conference Series 110, eds H. R. Miller, J. R. Webb, \& J. C. Noble (San Francisco: ASP), 17

Padovani, P., \& Giommi, P. 1995, MNRAS, 277, 1477

Qian, S. J., et al. 1991, A\&A, 241, 15

Romero, G. E., Cellone, S. A., \& Combi, J. A. 1999, A\&AS, 135,477

Schmidt, M., \& Green, R. F. 1983, ApJ, 269, 352

Shen, Z., et al. 1998, AJ, 115, 1357

Siebert, J., et al. 1998, MNRAS, 301, 261

Tadhunter, C. N., et al. 1993, MNRAS, 263, 999

Wagner, S. J., \& Witzel, A. 1995, ARA\&A, 33, 163

Whiting, M. T., Webster, R. L., \& Francis, P. J. 2001, MNRAS, 323, 718 (WWF)

Wills, B. J., et al. 1992, ApJ, 398, 454 